\title{
Caracterización de la familia del anciano con deterioro cognitivo
}

\author{
Characterization of the family of old people with cognitive \\ impairment
}

\author{
Dra. Marcia Maritza Ávila Oliva \\ Policlínico Universitário "Alcides Pino Bermúdez". Holguín, Cuba.
}

\section{RESUMEN}

Introducción: con la prolongación de la esperanza de vida ha aumentado la incidencia y prevalencia de enfermedades que aparecen en edades avanzadas, entre ellas las demencias.

Objetivo: caracterizar a las familias de adultos mayores con deterioro cognitivo según variables de interés.

Métodos: estudio descriptivo de corte transversal en familias de adultos mayores con deterioro cognitivo pertenecientes al Consejo Popular "Alcides Pino" del municipio Holguín en el año 2010. Se trabajó con 139 familias identificadas por el geriatra o por los médicos de consultorios. Para la recogida de la información, se utilizaron la entrevista y el análisis documental y se aplicó un instrumento específico para clasificar a las familias según su funcionamiento.

Resultados: se encontró que el $41,6 \%$ eran familias ampliadas y el $38,8 \%$ extensa, el $36,1 \%$ y el 30,6 \% estaban en etapa de contracción y disolución, respectivamente. Todas las familias tenían crisis familiares con predominio de las paranormativas en el $86,1 \%$. El afrontamiento a la enfermedad del anciano fue inadecuado en el $88,9 \%$ de las familias y el $38,9 \%$ eran disfuncionales.

Conclusiones: las familias del Consejo Popular "Alcides Pino", que conviven con adultos mayores con deterioro cognitivo están muy lejos de ser la red de apoyo que necesitan estas personas en el proceso de su enfermedad.

Palabras clave: Familia, deterioro cognitivo, crisis familiares, afrontamiento. 


\section{ABSTRACT}

Introduction: the extension of life expectancy has increased the incidence and prevalence of diseases that occur at older ages such as dementias.

Objective: to characterize the families of the older adults with cognitive impairment, according to variables of interest.

Methods: cross-sectional descriptive study conducted on families of older adults with cognitive impairment in "Alcides Ríos" people's council located in "Holguín" municipality during 2010. The sample was made up of 139 families identified by the geriatricians or by the family physicians. For data collection, the interviews and the documentary review were used together with a customized instrument to classify families on the basis of their functioning.

Results: it was found that $41.6 \%$ of the sample was extended families and $38.8 \%$ corresponded to big families; $36.1 \%$ and $30.6 \%$ of them were getting smaller or were vanishing respectively. All these families faced some family crisis with paranormative crises in $86.1 \%$ of cases. Inadequate management of the old person's disease was seen in $88.9 \%$ of families whereas $38.9 \%$ of them were dysfunctional. Conclusions: the families of older people with cognitive impairment in "Alcides Pino" people's council were far from being the adequate supporting network required by these elders.

Key words: Family, cognitive impairment, family crisis, confrontation.

\section{INTRODUCCIÓN}

En los países desarrollados, la enfermedad de Alzheimer es la tercera enfermedad en costos sociales, después de las enfermedades cardiacas y el cáncer. Sin embargo, el mayor costo de esta enfermedad es, sin lugar a dudas, su costo humano, cuyas dimensiones son inestimables, no solo para los enfermos sino para la familia. ${ }^{1,2}$

La familia ocupa un lugar fundamental en la formación de motivos y comportamientos implicados en la salud y su protección, en el desencadenamiento y recuperación de la enfermedad, en las decisiones sobre el uso de servicios profesionales y constituye la red de apoyo más potente y eficaz en el ajuste a la vida social y muy en especial ante los procesos de salud, enfermedad y muerte.

El objetivo que guía este estudio es caracterizar a las familias de adultos mayores con deterioro cognitivo según su composición, etapa del ciclo vital en la que se encontraban en el período del estudio, tipo de crisis presentes, comportamiento según su afrontamiento a la enfermedad del adulto mayor y su funcionamiento.

\section{MÉTODOS}

Se realizó un estudio descriptivo transversal en familias de adultos mayores con deterioro cognitivo pertenecientes al Consejo Popular "Alcides Pino" del área de salud del mismo nombre del municipio de Holguín en el año 2010. Como parte de un proyecto social que está teniendo lugar en este Consejo Popular, de un universo

http://scielo.sld.cu 
de 142 familias de adultos mayores con deterioro cognitivo, se trabajó con una muestra de 139 las cuales fueron identificadas por el geriatra o por los médicos de consultorios y que cumplieron con los siguientes criterios de inclusión:

- Familias donde el cuidador principal no presentara déficit sensorial que interfiriera en la comunicación y que su estado mental garantizara la confiabilidad de los datos aportados.

- Familia que diera su consentimiento a participar en el estudio.

Las familias se clasificaron según su composición y teniendo en cuenta su ontogénesis, en familia nuclear, extensa y ampliada. ${ }^{3}$

Familia nuclear: aquella que está integrada por una pareja que tenga hijos o no, o por uno de los miembros de la pareja con su descendencia. Incluye los hijos sin padres en el hogar, los hijos de uniones anteriores, la adopción y el equivalente de pareja.

Familia extensa: aquella que desciende de un mismo tronco, independientemente del número de generaciones y que está integrada por una pareja con hijos cuando al menos uno de ellos convive en el hogar con su pareja, o equivalente de pareja, con descendencia o sin ella.

Familia ampliada: cuando a la familia nuclear o extensa se integran otros parientes que no pertenecen al mismo tronco de descendencia generacional.

Se pueden considerar otros casos en los que aunque no existan vínculos consanguíneos y de parentesco entre ellos, sí existen de convivencia y afinidad.

Para identificar la etapa del ciclo vital en la que se encontraban las familias, se tuvo en cuenta: etapa de formación, desde el matrimonio hasta el nacimiento del primer hijo; etapa de extensión, desde el nacimiento del primer hijo hasta que el primer hijo sale del hogar; etapa de contracción, desde que el primer hijo sale del hogar hasta la muerte del primer cónyuge; etapa de disolución, desde la muerte del primer cónyuge hasta la muerte del segundo cónyuge. ${ }^{3}$

Para identificar el tipo de crisis presentes en las familias, se consideraron crisis transitorias o normativas, aquellas que dependen del tránsito de una etapa a otra del ciclo y del enfrentamiento a los acontecimientos en el proceso de desarrollo de la familia; crisis no transitorias o paranormativas, aquellas derivadas de los acontecimientos accidentales que pueden ocurrir en cualquier etapa del ciclo y afectan de manera muy variable en dependencia de las características y principios de cada familia. ${ }^{3}$

El afrontamiento a la enfermedad, se consideró adecuado cuando la familia es capaz de asumir y enfrentar la situación que presenta y busca ayuda si lo considera necesario, por lo contrario, se consideró inadecuado cuando la familia no enfrenta esta situación y crea obstáculos que impiden la ayuda externa.

El funcionamiento familiar se evaluó mediante la aplicación de un instrumento diseñado en Cuba para estos fines y que tiene en cuenta cómo se da la dinámica relacional sistémica entre los miembros de la familia. Se denomina Prueba de Funcionamiento Familiar (FF-SIL) y consta de 14 proposiciones y 7 categorías. ${ }^{4}$ 


\section{RESULTADOS}

Al realizar la distribución de las familias de adultos mayores con deterioro cognitivo según su composición (tabla 1 ) se obtuvo que de las 139 familias predominaran las ampliadas para el $41,0 \%$, solo el $22,3 \%$ eran nucleares.

Tabla 1. Distribución de las familias según composición. Área de salud "Alcides Pino", 2010

\begin{tabular}{|c|c|c|}
\hline $\begin{array}{l}\text { Composición } \\
\text { de la familia }\end{array}$ & No. & $\%$ \\
\hline Nuclear & 31 & 22,3 \\
\hline Extensa & 51 & 36,7 \\
\hline Ampliada & 57 & 41,0 \\
\hline Total & 139 & 100,0 \\
\hline
\end{tabular}

Fuente: historias clínicas.

En relación con el ciclo vital de las familias en estudio (tabla 2), se notó que los mayores porcentajes correspondieron a las familias en estado de contracción y disolución, con el 35,3 y 29,5\%, respectivamente.

Tabla 2. Distribución de las familias según ciclo vital. Área de salud "Alcides Pino", 2010

\begin{tabular}{|l|r|c|}
\hline Ciclo vital de la familia & No. & $\%$ \\
\hline Formación & 19 & 13,6 \\
\hline \hline Extensión & 30 & 21,6 \\
\hline Contracción & 49 & 35,3 \\
\hline Disolución & 41 & 29,5 \\
\hline Total & 139 & 100,0 \\
\hline
\end{tabular}

Fuente: historias clínicas.

Resultó de interés conocer que todas las familias tenían crisis en el momento del estudio y que en 120 de ellas $(86,3 \%)$, eran de tipo no transitoria o paranormativa y en 73 familias $(52,5 \%)$, eran de tipo transitoria o normativa.

El afrontamiento a la enfermedad del adulto mayor por parte de la familia resultó inadecuado en 123 de ellas para el $88,5 \%$ y solo en 16 familias, para el $11,5 \%$, fue valorado como adecuado.

El $38,8 \%$ de las familias tenían un funcionamiento disfuncional y el $13,7 \%$ era severamente disfuncional (tabla 3 ).

http://scielo.sld.cu 
Tabla 3. Distribución de las familias según funcionamiento. Área de salud "Alcides Pino", 2010

\begin{tabular}{|l|c|c|}
\hline Funcionamiento familiar & No. & $\%$ \\
\hline Funcional & 24 & 17,2 \\
\hline Moderadamente funcional & 42 & 30,3 \\
\hline Disfuncional & 54 & 38,8 \\
\hline Severamente disfuncional & 19 & 13,7 \\
\hline Total & 139 & 100,0 \\
\hline
\end{tabular}

Fuente: historias clínicas. Clasificación FF-SIL.

\section{DISCUSIÓN}

El Consejo Popular donde se realizó el estudio, presenta características sociodemográficas favorecedoras para el comportamiento familiar descrito, pues por situaciones de disponibilidad de viviendas en su mayoría o por ayuda mutua familiar, en el menor de los casos, coexisten dos o más familias nucleares compartiendo el mismo hogar formándose familias extensas y ampliadas. En un estudio realizado en el área de salud del policlínico "Dr. Manuel Fajardo Rivero" del municipio Playa, ubicado en La Habana, predominaron las familias compuestas por tres generaciones. ${ }^{5}$ En otros estudios realizados en familias cubanas han predominado las familias multigeneracionales. ${ }^{3,6}$

La existencia de hogares multigeneracionales, como se señaló anteriormente, favorece la coexistencia de funciones, tareas y conflictos derivados de varias etapas del ciclo vital, de acuerdo a los eventos de vida que se presentan.

Cuando existen crisis en la familia se produce un proceso de agudización de contradicciones internas familiares que ponen de manifiesto la necesidad de un ajuste de las relaciones, en función de las demandas provenientes del propio medio interno familiar y de su relación con el medio externo. ${ }^{7}$

En el proceso de desarrollo de una familia cualquiera, se constatan períodos de estabilidad y otros de agudas contradicciones, algunas propias del desarrollo de los miembros en su ciclo de vida individual, que repercuten en el sistema familiar y otras contradicciones que reflejan ciertas derivaciones del proceso normal de desarrollo y asimilación social. ${ }^{7}$ Cualquier familia puede atravesar a la vez dos o tres tipos de crisis. La presencia de crisis no quiere decir que la familia se esté deteriorando sino que estas son motores impulsores de los cambios. Ambos tipos de tareas requieren en gran medida de la capacidad de adaptación, ajuste y equilibrio de la familia para que sea capaz de desarrollarse y enfrentar los momentos críticos de una manera adecuada y mantener el equilibrio psicológico de sus miembros. En estudio realizado en un área de salud del municipio Playa, La Habana, las crisis de más alta tasa de aparición fueron la de desmembramiento, seguida de las de desorganización. ${ }^{8}$ Según Ares Muzio se puede distinguir que una de cada tres familias ha sufrido crisis de desorganización (Ares Muzio P. Mito o realidad. Algunas consideraciones para el estudio de la familia como objeto de investigación científica. La Habana, junio de 1995). 
La presencia de crisis en la familia, ya sea por el paso de una etapa a otra o derivadas de acontecimientos accidentales, depende en gran medida de cómo la familia es capaz de enfrentarlas. En el caso particular de este estudio, la presencia de un miembro de la familia con algún grado de deterioro cognitivo creó un desajuste en el equilibrio armónico de los integrantes del núcleo familiar, lo que conllevó a su desestabilización psicológica, resultados estos que coincidieron con los de otros autores. ${ }^{8,9}$

La familia con un buen afrontamiento busca ayuda en otros parientes, amistades, maestros y en otros profesionales cuando considera que sus problemas desbordan sus propios recursos, sin embargo, hay familias que desarrollan mecanismos de barreras frente a la ayuda profesional, se niegan a seguir orientaciones especializadas, desestiman la ayuda externa y evaden enfrentar cambios. ${ }^{4}$

En el caso del afrontamiento a situaciones de salud, existen familias que aplazan o no cumplen las indicaciones familiares para mejorar el estado de salud de alguno de los integrantes, niegan el diagnóstico y hasta rehúsan enfrentar las acciones propias de determinado tratamiento. Tal comportamiento potencializa su acción destructiva cuando se trata de menores, ancianos o discapacitados que dependen totalmente de las decisiones que sus familiares tomen por ellos. ${ }^{4}$

La familia en crisis no es necesariamente una familia disfuncional o con problema. Si la familia establece estrategias disfuncionales ante las situaciones de cambio, como la rigidez y la resistencia, esto provoca un enquistamiento de los conflictos y por tanto, comienzan a aparecer síntomas que atentan contra la salud y el desarrollo armónico de sus miembros. Por lo tanto, la principal característica que debe tener una familia funcional es que promueva un desarrollo favorable a la salud para todos sus miembros, para lo cual es imprescindible que tenga: jerarquías claras, límites claros, roles claros y definidos, comunicación abierta y explícita y capacidad de adaptación al cambio.

La presencia de crisis no necesariamente es la causa de disfunción familiar sino el modo en que la enfrenta. Walsh y Chagoya consideran que las familias funcionales encaran las crisis como grupo, buscando solución adecuada a los conflictos, cada miembro acepta un papel para hacerles frente, identifican los problemas expresando conformidades e inconformidades y se respeta la autonomía a la vez que los espacios y los roles están bien definidos. ${ }^{4}$

La familia funcional cumple con la misión social sin afectar el proceso interno de interacción, la calidad de las relaciones internas y el desarrollo individual según los requerimientos de cada etapa. ${ }^{4}$

Estas familias son capaces de abandonar las conductas que les han resultado ineficaces y se formulan creativamente nuevas estrategias, tienen un desempeño alto en la resolución de problemas con eficacia.

Las familias disfuncionales son incapaces de enfrentar las crisis, expresar con libertad los sentimientos negativos, no identifican los problemas, no se comunican con claridad, utilizan dobles mensajes, entorpecen el desarrollo individual y existe confusión de roles. Repiten las conductas ineficaces y culpan a otros con reacciones emocionales excesivas o simplemente niegan la existencia del problema. Las soluciones que adoptan pasan a ser el verdadero problema. Se observa en ellas conflictos de roles, alteración de la organización jerárquica, no disponen de capacidad para negociar sus discrepancias y se muestran intolerantes ante las opiniones de los otros. De igual forma se hacen culpables de sus fracasos y se cohíben de expresar sus emociones ante los miembros de la familia. ${ }^{4}$ 
En este estudio predominaron las familias disfuncionales, lo que coincide con los resultados de la investigación realizada en el municipio Playa, La Habana, anteriormente mencionada. ${ }^{8}$

Se puede concluir que las familias del Consejo Popular "Alcides Pino", que conviven con adultos mayores con deterioro cognitivo están muy lejos de constituirse en la red de apoyo que necesitan estas personas en el proceso de su enfermedad.

\section{REFERENCIAS BIBLIOGRÁFICAS}

1. Llibre Guerra JC, Guerra Hernández MA, Perera Miniet E. Impacto psicosocial del síndrome demencial en cuidadores cruciales. Rev Cubana Med Gen Integr

[Internet]. 2008 [citado 29 Dic 2010];24(1). Disponible en: http://scielo.sld.cu/scielo.php?script=sci arttext\&pid=S0864 $\underline{21252008000100005 \& \operatorname{lng}=e s \& n r m=i s o \& \text { tlng=es }}$

2. Llibre Rodríguez J, Guerra Hernández M. Enfermedad de Alzheimer. Situación actual y estrategias terapéuticas. Rev Cubana Med. 1999;38(2):134-42.

3. Louro I. Atención Familiar. En: Álvarez Sintes, editor. Temas de Medicina General Integral. Salud y Medicina. La Habana: Editorial Ciencias Médicas; 2000. p. 209-38.

4. Louro Bernal I. Modelo teórico-metodológico para la evaluación de salud del grupo familiar en la atención primaria [tesis]. La Habana: Escuela Nacional de Salud Pública; 2004.

5. Almenares Aleaga M, Louro Bernal I, Ortiz Gómez MT. Comportamiento de La violencia intrafamiliar. Rev Cubana Med Gen Integr. 1999;15(3).

6. Pérez Roja N. Características sociodemográficas de la familia cubana, 19531970. La Habana: Editorial Ciencias Sociales; 1979.

7. Sosa L. Características psicosociales de las familias de un consultorio médico [tesis]. La Habana: Facultad de Salud Pública; 1995.

8. Ortiz Gómez MT, Louro Bernal I, Jiménez Cangas L, Silva Ayçaguer LC. Salud familiar, caracterización en un área de salud. Rev Cubana Med Gen Integr

[Internet]. 1999 [citado 29 Dic 2010];15(3). Disponible en:

http://scielo.sld.cu/scielo.php?script=sci arttext\&pid=S0864 -

21251999000300014\&lng=es\&nrm=iso\&tlng=es

9. Pérez Martínez VT, Lorenzo Parra Z. Impacto del déficit mental en el ámbito familiar. Rev Cubana Med Gen Integr [Internet]. 2007 [citado 29 Dic 2010];23(3). Disponible en: http://scielo.sld.cu/scielo.php?script=sci arttext\&pid=S0864$21252007000300002 \& \operatorname{lng}=\mathrm{es} \& \mathrm{nrm}=\mathrm{iso} \& \mathrm{t} \operatorname{lng}=\mathrm{es}$

Recibido: 31 de marzo de 2011.

Aprobado: 9 de noviembre de 2011.

Marcia Maritza Ávila Oliva. Prolongación de Narciso López CMF No. 35 e/ Benjamín de Zayas y Rosalía Fernández. Reparto Zayas. Holguín, Cuba. Teléfono: 42-7456. Correo electrónico: marcia@cristal.hlg.sld.cu 